\title{
A Comparative Review of Demographics, Incidence, and Epidemiology of Histologically Confirmed Intracranial Tumors in Brazil and Bulgaria
}

George S. Stoyanov ${ }^{1}$, Jonathan S. Sarraf ${ }^{2}$, Boyko K. Matev ${ }^{3}$, Deyan L. Dzhenkov ${ }^{4}$, Martina Kitanova ${ }^{5}$, Bogomil Iliev ${ }^{6}$, Peter Ghenev ${ }^{7}$, Anton B. Tonchev ${ }^{8}$, Yavor Enchev ${ }^{6}$, Fernando Adami $^{9}$, Luis Eduardo W. De Carvalho ${ }^{9}$

1. General and Clinical Pathology/Forensic Medicine and Deontology, Medical University, Varna, BGR 2. Genetic and Molecular Biology, Universidade Federal Do Pará, Belém, Pará, Brazil 3. Medicine, Medical University, Varna, BGR 4. General and Clinical Pathology, Forensic Medicine and Deontology, Medical University, Varna, BGR 5. Department of General and Clinical Pathology, Forensic Medicine and Deontology, Faculty of Medicine, Medical University - Varna “Prof. Dr. Paraskev Stoyanov”, Varna, Bulgaria 6. Department of Neurosurgery and Ent Diseases, Division of Neurosurgery, Faculty of Medicine, Medical University - Varna “Prof. Dr. Paraskev Stoyanov", Varna, Bulgaria 7. Department of General and Clinical Pathology, Forensic Medicine and Deontology, Faculty of Medicine, Medical University - Dr. Paraskev Stoyanov, Varna, BGR 8. Department of Anatomy and Cell Biology, Faculty of Medicine, Medical University - Varna “Prof. Dr. Paraskev Stoyanov”, Varna, Bulgaria 9. Laboratory of Epidemiology and Data Analysis, Faculdadede Medicina Do Abc, Santo André, São Paulo, Brazil

$\square$ Corresponding author: George S. Stoyanov, georgi.geesh@gmail.com Disclosures can be found in Additional Information at the end of the article

Received 02/06/2018 Review began 02/09/2018 Review ended 02/12/2018 Published 02/19/2018

\section{(C) Copyright 2018}

Stoyanov et al. This is an open access article distributed under the terms of the Creative Commons Attribution License CC-BY 3.0., which permits unrestricted use, distribution, and reproduction in any medium, provided the original author and source are credited.

\section{Abstract}

Intracranial tumors (ICTs) attract numerous scientific teams and tremendous financial resources worldwide. These lesions of the central nervous system (CNS) can be both benign and malignant in biological behavior as well as local or metastatic in origin. We compared data from two studies on primary and metastatic ICTs from Brazil and Bulgaria, based on histopathologically confirmed ICTs from tertiary health centers. Primary ICTs significantly outweigh the frequency of metastatic ICTs. Primary ICTs represent $86.45 \%$ in Brazil and 69.17\% in Bulgaria, with around $60 \%$ of their totals being malignant. There is a statistical dominance of tumors from the neuroepithelial origin, with the most common entry being glioblastoma multiforme. The second-most common primary ICT group comprises tumors of meningeal origin. Metastatic ICTs show great variance; 13.55\% in Brazil and 31.38\% in Bulgaria of all ICT cases being attributed to them. However, metastatic ICTs are even a more diverse group than neuroepithelial tumors, with the majority of this group comprising metastatic colorectal adenocarcinoma (almost exclusively in males), metastatic breast adenocarcinoma in females, metastatic pulmonary carcinomas (primarily from the non-small cell group with a male predominance), and metastatic melanoma with an even gender ratio.

Categories: Neurology, Neurosurgery, Oncology

Keywords: primary intracranial tumors, metastatic intracranial tumors, incidence, frequency, ethnic factors, demographics, pathology, oncology, statistics, comparison

\section{Introduction And Background}

Nowadays the scientific research of intracranial tumors (ICTs) attracts numerous scientific teams and tremendous financial resources worldwide [1-3]. These lesions of the central nervous 
system (CNS) and structures located in the cranial cavity can be both benign and malignant in biological behavior as well as local or metastatic in origin for malignant lesions [4-6]. They are rarely diagnosed in their early stages of biological development, require highly trained multidisciplinary medical team for their diagnosis and treatment, have a high impact on the patients and their relative quality of life, with some primary and metastatic entries having a very dismal survival prognosis, together with a high resource impact on the medical system [7$16]$.

Direct comparisons of primary and metastatic ICTs are very rare as they require a complex methodology of case gathering and calculation. Due to these exact reasons, comparative studies of primary and metastasic ICTs have not been carried out since the mid-1980s [17-19]. Since then, the standard has been that studies of primary CNS tumors must always apply to pathological criteria, while metastatic ICTs may only adhere to the radiological findings [2032].

The aim of this review is to discuss our experiences and compare the findings regarding ICTs in our distinct populations, based on our data from Brazil, published by de Carvalho, et al. spanning across 17 years and 949 cases and from Bulgaria, published by Stoyanov, et al. spanning across five years and 798 cases [33-34].

\section{Review}

The data was compared on the merit of major histopathological groups to accommodate the differences in the 2006 and 2016 World Health Organization (WHO) classification of tumors of the CNS intracranial tumors used in the two separate studies (Table 1) [6, 33-35]. Metastatic tumors were considered those not arising from the cranial cavity and the structures inside it, including infiltrative tumors such as lymphomas and leukemias. Lymphomas were compared based on their location of origin and systemic presentation, to conclude their origin as either primary CNS lymphoma or infiltrative CNS lymphomas. 


\section{Cureus}

\section{Variable}

\section{Gender}

Male

Female

Histological type

Tumors of Neuroepithelial tissue

Tumours of the meninges

Tumors of cranial and paraspinal nerves

Lymphomas and haematopoietic neoplasms

Germ cell tumors

Tumors of the sellar region

Metastatic tumours

Others

Total

Age group

$<20$ years

20-39 years

40-59 years

$>60$ years

Median age

\section{Brazil}

N

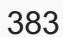

414

48.06

558

51.94

240

30.08

40.90

248

31.08

172

21.28

213

26.69

50

6.27

12

1.51

10

1.25

7

0.88

9

1.13

8

1

$\begin{array}{llll}80 & 10.04 & 46 & 5.76\end{array}$

108

13.55

254

31.83

42

5.27

10

1.25

797

100

798

100

199

190

254

154

40
24.96

23.81

31.86

19.33

199

50

TABLE 1: Comparison between ICTs in Brazil and Bulgaria with regards to gender, main histopathological group, age groups and median age

ICT: intracranial tumor

Due to the different approaches, data gathering and WHO classifications used in the separate studies, 797 cases from a total of 1027 were used from the Brazilian population and 798 cases from a total of 822 were used from the Bulgarian population, accounting for a total of 1,595 ICT cases from both populations (Table 1). Both studies were based on collection of histopathologically confirmed specimens from tertiary health centers.

In both cohorts, primary ICTs significantly outweigh the frequency of metastatic ICTs [33-34]. 


\section{Cureus}

Primary ICTs represent $86.45 \%$ in Brazil and 69.17\% in Bulgaria of all ICTs, based on the histological verification criteria, with around $60 \%$ their total being malignant in nature (Figure $1)$.

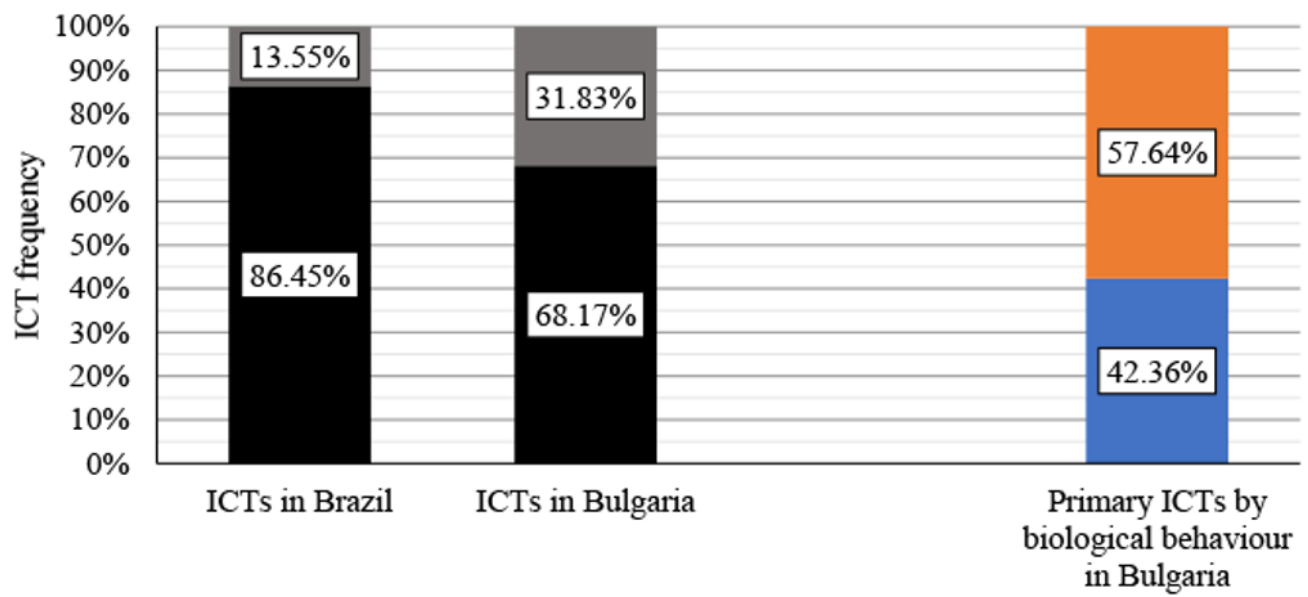

- Primary ICTs $\quad$ Metastatic ICTs $\quad$ Benign primary ICTs $=$ Malignant primary ICTs

FIGURE 1: Comparison between ICTs in Brazil in Bulgaria, with benign and malignant comparison for primary ICTs in Bulgaria

ICT: intracranial tumor

Both studies found a statistical dominance of tumors from the neuroepithelial origin, with the most common entries being glioblastoma multiforme, with other astrocytic and glial tumors accommodating for a relatively small number of cases [33-34]. These results confirm other large case number reports [24-26].

The second most common primary ICT group is that of the tumors of meningeal origin, which are close to the total frequency of the very diverse group of neuroepithelial tumors (Table 1). However, since the major entry in this group are meningiomas, with some vascular and other rare entries accounting for a very small number of cases, meningiomas are statistically the most common ICT tumor type [33-34].

ICT groups such as primary lymphomas of the CNS, germ cell tumors and nerve sheath tumors account for a total of around $10 \%$ of all histologically confirmed cases of ICTs (Table 1). Tumors of the sellar region, despite being very common, clinically account for between five and $10 \%$ of all histologically confirmed cases of ICTs.

Metastatic ICTs are another big group, showing great variance in the two populations with 13.55\% in Brazil and 31.38\% in Bulgaria of all ICT cases being attributed to them (Table 1). However, metastatic ICTs are even a more diverse group than neuroepithelial tumors, with the majority of this group being due to metastatic colorectal adenocarcinoma (diagnosed almost exclusively in males), metastatic breast adenocarcinoma (diagnosed in females), metastatic pulmonary carcinomas primarily from the non-small cell group with a male predominance and metastatic melanoma with an even male to female ratio [33-34]. Some of the differences in the group of metastatic ICTs may be attributed to the variance of mutations described in the populations [36]. 
Other extremely rare entries such as pineal tumors, myofibroblastic tumors, and others can contribute to an aggregated total of five percent of histologically confirmed ICTs (Table 1).

Regarding metastatic location in the Bulgarian population, only $12.20 \%$ of cases of metastatic ICTs were found in the subtentorial space. There were no metastatic ICTs with predominant subtentorial metastatic space.

Despite the similarities of the histological findings, there are some visible differences in the results; in Brazil, the most affected age group is 40-59 years, followed by 0-19 years, whilst in Bulgaria, the most affected age group is $40-59$ years, followed by $>60$ years (Table 1 ). These differences may be attributed to the significantly higher number of metastatic ICTs in the Bulgarian population when compared to the Brazilian population. Despite this, both studies describe a major peak of incidence in the age group 40-59 years when compared to the other age groups [33-34].

Despite the complexity of the separate groups of ICTs and the ethnic differences of both populations, ICTs remain an underestimated group of tumors with primary ICTs accounting for more than $2 \%$ of all newly diagnosed malignancies per year [37]. When adding the data of nonmalignant primary ICTs and metastatic ICTs, the total incidence increases to around $4 \%$ of all cancer cases, equal to that of cancer types with which the population and general medical peers are much better acquainted, such as head and neck malignancies [38].

According to data collected from the Bulgarian population, the annual incidence of ICTs is 8.85 people per 100,000, comprised of 6.03 per 100,000 for primary ICTs and 2.82 for metastatic ICTs [34]. Furthermore, in other European populations, the reported incidence is even higher than the reported figures in our studies [3].

Although significantly more common than primary ICTs, metastatic ICTs represent only a small part of surgical specimens, as most patients with metastatic ICTs are either inoperable, suitable for radiosurgery only, or otherwise considered terminal.

The problematic of systemic analyses of ICTs demand a very strict choice of methods, as the different entries require different approaches to diagnosis. Although all tumors should be verified pathohistologically for the diagnosis to be certain, in the case of metastatic ICTs, this is seldom the case as they are often only diagnosed on clinical or radiological data alone. This choice of clinical methodological approach does not certify the tumor type, as the chance of a distant malignant entry, albeit often metastasizing to the CNS, does not exclude the presence of synchronous CNS tumor [31, 39].

Such cases are however very rare and most commonly presented only as a case report and case series and individual approaches are more often than not taken as absolute. This is due to combined pathohistological and radiological criteria that would severely narrow down the study cohort and almost always report the same findings as the pathological criteria alone, while any cases that would be proven otherwise by pathology would only fit in the statistical error part of a radiological study. A further option on the matter is the rarely undertaken autopsy finding study as it reports on a very small series of cases, even in a greater timeframe [40].

Despite differences in some aspects of the individual study designs, the reported results are quite similar and a further representation of the adequacy of the individually given data. If taken together, the data volume spans across a total of 22 calendar years and 1595 cases and is the most complete representation of primary and malignant tumors of the CNS up to date, outweighing similar studies carried out in the past, by a great number of cases [17-18]. 
Furthermore, both studies report a greater number of cases and further increase the statistical understanding and epidemiological data of CNS tumors such as de Carvalho, et al. which also depicts CNS spinal tumors, whilst Stoyanov, et al. depicts non-tumor volume-occupying lesions such as cysts and abscesses with a total annual incidence of 0.27 people per 100,000 .

As seen in a direct comparison of the individual data, the findings are similar in a number of categories, despite some visible differences, primarily in median age and total male to female ratio. Still, the findings are not that dissimilar when compared to individual reports on primary or metastatic ICTs alone [20-21, 24-26]. This is both due to the pathological criteria of both studies and the large number of patients reported in each study.

One of the most intriguing parts of the comparison of results is the wide similarity of the results, despite the significant ethnic differences of the populations, questioning the impact of ethnic factors in CNS tumors pathobiology as described in previous studies [41-42].

\section{Conclusions}

Although significantly more common than primary ICTs, metastatic ICTs represent only a small part of surgical and histopathological specimens, as most patients with metastasic ICTs are either inoperable, suitable for radiosurgery only, or otherwise considered terminal.

The demographic data represented in both studies demonstrate a severe difference in age groups on the background of the similar histological findings, which may be the only ethnic aspect confirmed by the reports, this time confirming the demographic specifics of ICTs.

Despite all the individually reported results and the lesser percentage of metastatic ICTs, both sets of authors agree that the pathological verification criteria do not report the total incidence of metastatic ICTs, which should, in total, given the globally increasing reported incidence of cancer, outweigh the percentage of primary ICTs.

\section{Additional Information \\ Disclosures}

Conflicts of interest: In compliance with the ICMJE uniform disclosure form, all authors declare the following: Payment/services info: All authors have declared that no financial support was received from any organization for the submitted work. Financial relationships: All authors have declared that they have no financial relationships at present or within the previous three years with any organizations that might have an interest in the submitted work. Other relationships: All authors have declared that there are no other relationships or activities that could appear to have influenced the submitted work.

\section{Acknowledgements}

George S Stoyanov and Jonathan S Sarraf contributed equally to this work.

\section{References}

1. Stoyanov GS, Dzhenkov DL: On the concepts and history of glioblastoma multi-forme morphology, genetics and epigenetics. Folia Med (Plovdiv). 2018, 60:ahead of print.

2. Michaud K, de Tayrac M, D'Astous M, et al.: Contribution of 1p 19q, 9p and 10q automated analysis by FISH to the diagnosis and prognosis of oligodendroglial tumors according to WHO 2016 guidelines. PLoS One. 2016, 11 :e0168728. Accessed: February 18, 2018: 10.1371/journal.pone.0168728

3. Fuentes-Raspall R, Solans M, Roca-Barceló A, et al.: Descriptive epidemiology of primary 
malignant and non-malignant central nervous tumors in Spain: Results from the Girona Cancer Registry (1994-2013). Cancer Epidemiol. 2017, 50:1-8. 10.1016/j.canep.2017.07.005

4. Nguyen LN, Maor MH, Oswald MJ: Brain metastases as the only manifestation of an undetected primary tumor. Cancer. 1998, 83:2181-4. 10.1002/(SICI)1097-

0142(19981115)83:10<2181::AID-CNCR17>3.0.CO;2-J

5. Gavrilovic IT, Posner JB : Brain metastases: epidemiology and pathophysiology. J Neurooncol. 2005, 75:5-14. 10.1007/s11060-004-8093-6

6. Louis DN, Perry A, Reifenberger G, et al.: The 2016 World Health Organization classification of tumors of the central nervous system: a summary. Acta Neuropathol. 2016, 131:803-20. 10.1007/s00401-016-1545-1

7. Yang P, Zhang W, Wang Y, et al.: IDH mutation and MGMT promoter methylation in glioblastoma: results of a prospective registry. Oncotarget. 2015, 6:40896-906. Accessed: February 18, 2018: 10.18632/oncotarget.5683

8. Darefsky AS, King JT Jr, Dubrow R: Adult glioblastoma multiforme survival in the temozolomide era: a population-based analysis of Surveillance, Epidemiology, and End Results registries. Cancer. 2012, 118:2163-72. 10.1002/cncr.26494

9. Krex D, Klink B, Hartmann C, et al.: Long-term survival with glioblastoma multiforme . Brain. 2007, 130:2596-606. 10.1093/brain/awm204

10. Stoyanov GS, Dzhenkov Dl, Kitanova M, et al.: Correlation between Ki-67 index World Health Organization grade and patient survival in glial tumors with astrocytic differentiation.

Cureus. 2017, 9:e1396. Accessed: February 18, 2018: 10.7759/cureus.1396

11. Katzman GL, Dagher AP, Patronas NJ: Incidental findings on brain magnetic resonance imaging from 1000 asymptomatic volunteers. JAMA. 1999, 282:36-39. 10.1001/jama.282.1.36

12. Nakamura M, Roser F, Michel J, et al.: The natural history of incidental meningiomas. Neurosurgery. 2003, 53:62-71. 10.1227/01.NEU.0000068730.76856.58

13. Pallud J, Fontaine D, Duffau H, et al.: Natural history of incidental world health organization grade II gliomas. Ann Neurol. 2010, 68:727-33. 10.1002/ana.22106

14. Sands SA, Kellie SJ, Davidow AL, et al.: Long-term quality of life and neuropsychologic functioning for patients with CNS germ-cell tumors: from the First International CNS GermCell Tumor Study. Neuro Oncol. 2001, 3:174-83. 10.1093/neuonc/3.3.174

15. Stoyanov GS, Dzhenkov D, Ghenev P: Cytokeratin AE1/AE3 mimicry in glioblastoma. Scri Sci Med. 2017, 49:49-52. 10.14748/ssm.v49i1.2055

16. Stoyanov GS, Dzhenkov D, Ghenev P: The great imitator - EMA positive glioblastoma multiforme. Scri Sci Med. 2017, 49:21-5. 10.14748/ssm.v49i1.2056

17. Guomundsson KR: A survey of tumours of the central nervous system in Iceland during the 10-year period, 1954-1963. Acta Neurol Scand. 1970, 46:538-52. 10.1111/j.16000404.1970.tb05811.x

18. Walker AE, Robins M, Weinfeld FD: Epidemiology of brain tumors: the national survey of intracranial neoplasms. Neurology. 1985, 35:219-226. 10.1212/WNL.35.2.219

19. Fogelholm R, Uutela T, Murros K: Epidemiology of central nervous system neoplasms. A regional survey in Central Finland. Acta Neurol Scand. 1984, 69:129-36. 10.1111/j.16000404.1984.tb07791.x

20. Delattre JY, Krol G, Thaler HT, et al.: Distribution of brain metastases . Arch Neurol. 1988, 45:741-4. 10.1001/archneur.1988.00520310047016

21. Schouten LJ, Rutten J, Huveneers HA, et al.: Incidence of brain metastases in a cohort of patients with carcinoma of the breast, colon, kidney, and lung and melanoma. Cancer. 2002, 94:2698-705. 10.1002/cncr.10541

22. Kitajima K, Nakamoto Y, Okizuka H, et al.: Accuracy of whole-body FDG-PET/CT for detecting brain metastases from non-central nervous system tumors. Ann Nucl Med. 2008, 22:595-602. 10.1007/s12149-008-0145-0

23. Antoch G: Whole-body dual-modality PET/CT and whole-body MRI for tumor staging in oncology. JAMA. 2003, 290:3199-206. 10.1001/jama.290.24.3199

24. Ostrom QT, Gittleman H, Farah P, et al.: CBTRUS statistical report: Primary brain and central nervous system tumors diagnosed in the United States in 2006-2010. Neuro Oncol. 2013, 15:1-56. 10.1093/neuonc/not151

25. Ostrom QT, Gittleman H, Liao P, et al.: CBTRUS statistical report: primary brain and central nervous system tumors diagnosed in the United States in 2007-2011. Neuro Oncol. 2014, 16:1-63. 10.1093/neuonc/nou223 
26. Dolecek TA, Propp JM, Stroup NE, et al.: CBTRUS statistical report: primary brain and central nervous system tumors diagnosed in the United States in 2005-2009. Neuro Oncol. 2012, 14:1-49. 10.1093/neuonc/nos218

27. Surawicz TS, McCarthy BJ, Kupelian V, et al.: Descriptive epidemiology of primary brain and CNS tumors: results from the Central Brain Tumor Registry of the United States, 1990-1994. Neuro Oncol. 1999, 1:14-25. 10.1093/neuonc/1.1.14

28. Wrensch M, Minn Y, Chew T, et al.: Epidemiology of primary brain tumors: current concepts and review of the literature. Neuro Oncol. 2002, 4:278-99. 10.1093/neuonc/4.4.278

29. Fleury A, Menegoz F, Grosclaude P, et al.: Descriptive epidemiology of cerebral gliomas in France. Cancer. 1997, 79:1195-202. 10.1002/(SICI)1097-0142(19970315)79:6<1195::AIDCNCR19>3.0.CO;2-V

30. McNeill KA: Epidemiology of brain tumors. Neurol Clin. 2016, 34:981-998. 10.1016/j.ncl.2016.06.014

31. Nayak L, Lee EQ, Wen PY, et al.: Epidemiology of brain metastases. Curr Oncol Rep. 2012, 14:48-54. Accessed: February 18, 2018: 10.1007/s11912-011-0203-y

32. Smedby KE, Brandt L, Bäcklund ML, et al.: Brain metastases admissions in Sweden between 1987 and 2006. Br J Cancer. 2009, 101:1919-24. 10.1038/sj.bjc.6605373

33. de Carvalho LEW, Sarraf JS, Semblano AA: Central nervous system tumours profile at a referral center in the Brazilian Amazon region, 1997-2014. PLoS One. 2017, 12:e0174439. Accessed: February 18, 2018: 10.1371/journal.pone.0174439

34. Stoyanov GS, Dzhenkov DL, Kitanova M, et al.: Demographics and incidence of histologically confirmed intracranial tumors: a five-year, two-center prospective study. Cureus. 2016, 9:e1476. Accessed: February 18, 2018: 10.7759/cureus.1476

35. Louis DN, Ohgaki H, Wiestler OD, et al.: The 2007 WHO classification of tumours of the central nervous system. Acta Neuropathol. 2007, 114:97-109. 10.1007/s00401-007-0243-4

36. de Carvalho LEW, Sarraf JS, Oliveira ACM, et al.: What is different in the population of the Brazilian Amazon region so that they have a low frequency of KRAS gene mutations?. Case Rep Oncol. 2017, 10:777-82. 10.1159/000479733

37. Valerianova Z, Dimitrova N, Vukov M: Cancer Incidence In Bulgaria, 2013. Atanasov T (ed): Paradigma, Sofia; 2015.

38. Stoyanov GS, Kitanova M, Dzhenkov DL, et al.: Demographics of head and neck cancer patients: a single institution experience. Cureus. 2017, 9:e1418. Accessed: February 18, 2018: 10.7759/cureus.1418

39. LV M, Zhang X, Shen Y, et al.: Clinical analysis and prognosis of synchronous and metachronous multiple primary malignant tumors. Medicine (Baltimore). 2017, 96:e6799. Accessed: February 18, 2018: https://www.ncbi.nlm.nih.gov/pmc/articles/PMC5413286/

40. Tsukada Y, Fouad A, Pickren JW, et al.: Central nervous system metastasis from breast carcinoma. Autopsy study. Cancer. 1983, 52:2349-54. 10.1002/10970142(19831215)52:12<2349::AID-CNCR2820521231>3.0.CO;2-B

41. Abadin SS, Zoellner NL, Schaeffer M, et al.: Racial/ethnic differences in pediatric brain tumor diagnoses in patients with neurofibromatosis type 1. J Pediatr. 2015, 167:613-20. 10.1016/j.jpeds.2015.04.076

42. Gittleman H, Cote DJ, Ostrom QT: Do race and age vary in non-malignant central nervous system tumor incidences in the United States?. J Neurooncol. 2017, 134:269-77.

10.1007/s11060-017-2543-4 